${ }_{\text {LA-UR- }}^{96}-309 \underset{\text { Title: }}{4}$

W. C. Louis

$$
\text { CONF-9606284--! }
$$

LSND Neutrino Oscillation Results

\section{RECEIVED \\ SEP 231996 \\ OSTI}

VIIIth Rencontres de Blois

Neutrino: Dark Matter and the Universe

Blois, France

June 8-12, 1996

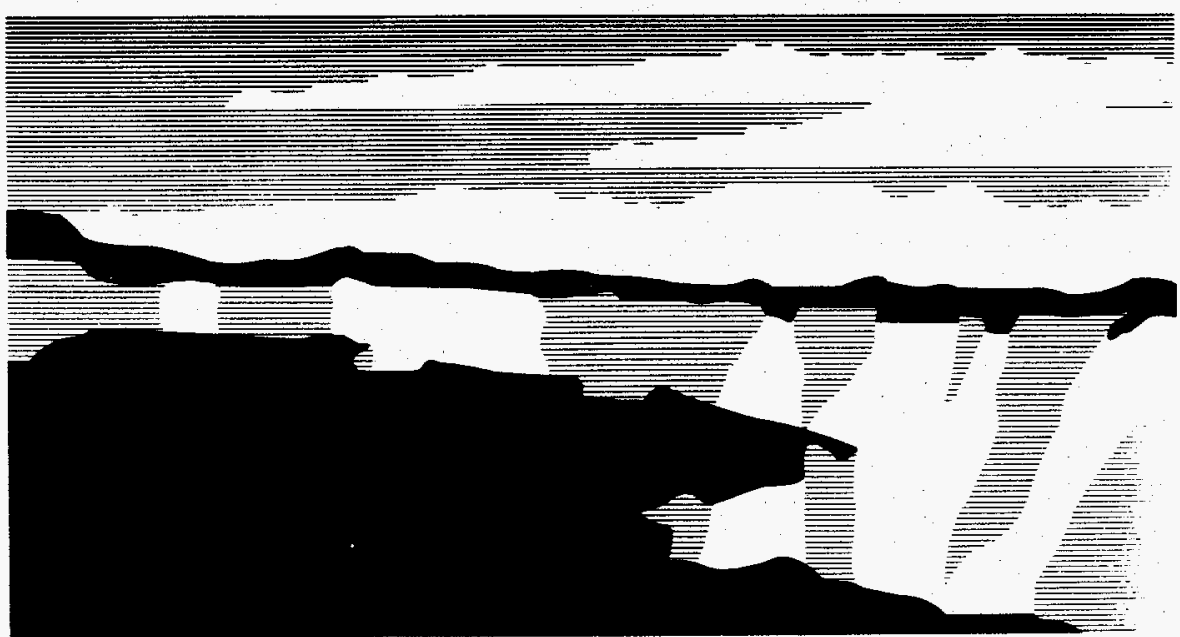

Los Alamos National Laboratory, an affirmative action/equal opportunity emplóyer, is operated by the University of California for the U.S. Department of Energy under contract W-7405-ENG-36. By acceptance of this article, the publisher recognizes that the U.S. Government retains a nonexclusive, royalty-free license to publish or reproduce the published form of this contribution, or to allow others to do so, for U.S. Government purposes. The Los Alamos National Laboratory requests that the publisher identity this article as work performed under the auspices of the U.S. Department of Energy. 


\section{DISCLAIMER}

Portions of this document may be illegible in electronic image products. Images are produced from the best available original document. 


\title{
LSND NEUTRINO OSCILLATION RESULTS
}

\author{
W. C. Louis, representing the LSND Collaboration 1) \\ Los Alamos National Laboratory, Los Alamos, NM 87545, USA
}

\section{DISCLAIMER}

\begin{abstract}
This report was prepared as an account of work sponsored by an agency of the United States Government. Neither the United States Government nor any agency thereof, nor any of their employees, makes any warranty, express or implied, or assumes any legal liability or responsibility for the accuracy, completeness, or usefulness of any information, apparatus, product, or process disclosed, or represents that its use would not infringe privately owned rights. Reference herein to any specific commercial product, process, or service by trade name, trademark, manufacturer, or otherwise does not necessarily constitute or imply its endorsement, recommendation, or favoring by the United States Government or any agency thereof. The views and opinions of authors expressed herein do not necessarily state or reflect those of the United States Government or any agency thereof.
\end{abstract}

\begin{abstract}
The LSND experiment at Los Alamos has conducted a search for $\bar{v}_{\mu} \rightarrow \bar{v}_{e}$ oscillations using $\bar{v}_{\mu}$ from $\mu^{+}$decay at rest. The $\bar{v}_{e}$ are detected via the reaction $\bar{v}_{e} p \rightarrow e^{+} n$, correlated with the 2.2-MeV $\gamma$ from $n p \rightarrow d \gamma$. The use of tight cuts to identify $e^{+}$ events with correlated $\gamma$ rays yields 22 events with $e^{+}$energy between 36 and $60 \mathrm{MeV}$ and only $4.6 \pm 0.6$ background events. The probability that this excess is due entirely to a statistical fluctuation is $4.1 \times 10^{-8}$. A $\chi^{2}$ fit to the entire $e^{+}$sample results in a total excess of $51.8_{-16.9}^{+18.7} \pm 8.0$ events with $e^{+}$energy between 20 and $60 \mathrm{MeV}$. If attributed to $\bar{v}_{\mu} \rightarrow \bar{v}_{e}$ oscillations, this corresponds to an oscillation probability (averaged over the experimental energy and spatial acceptance) of $(0.31 \pm 0.12 \pm 0.05) \%$.
\end{abstract}




\section{INTRODUCTION}

In the past several years, a number of experiments have searched for neutrino oscillations, where a neutrino of one type (say $\bar{v}_{\mu}$ ) spontaneously transforms into a neutrino of another type (say $\bar{v}_{e}$ ). For this phenomenon to occur, neutrinos must be massive and the apparent conservation law of lepton families must be violated. In 1995 the LSND experiment published data showing candidate events that are consistent with $\bar{v}_{\mu} \rightarrow \bar{v}_{e}$ oscillations. ${ }^{2)}$ Additional data are reported here that provide stronger evidence for neutrino oscillations. $\left.{ }^{3}\right)$

\section{DETECTOR}

The Liquid Scintillator Neutrino Detector (LSND) experiment at LAMPF 4) is designed to search with high sensitivity for $\bar{v}_{\mu} \rightarrow \bar{v}_{e}$ oscillations from $\mu^{+}$decay at rest. LAMPF is a most intense source of low energy neutrinos due to its $1-\mathrm{mA}$ proton intensity and $800-\mathrm{MeV}$ energy. The neutrino source is well understood 5) because almost all neutrinos arise from $\pi^{+}$or $\mu^{+}$decay; $\pi^{-}$and $\mu^{-}$are readily captured in the $\mathrm{Fe}$ of the shielding and $\mathrm{Cu}$ of the beam stop. The production of kaons and heavier mesons is negligible at these energies. The $\bar{v}_{c}$ rate is calculated to be only $4 \times 10^{-4}$ relative to $\bar{v}_{\mu}$ in the $36<E_{v}<52.8 \mathrm{MeV}$ energy range, so that the observation of a significant $\bar{v}_{e}$ rate would be evidence for $\bar{v}_{\mu} \rightarrow \bar{v}_{e}$ oscillations.

The LSND detector consists of an approximately cylindrical tank $8.3 \mathrm{~m}$ long by $5.7 \mathrm{~m}$ in diameter. The center of the detector is $30 \mathrm{~m}$ from the neutrino source. On the inside surface of the tank 1220 8-inch Hamamatsu phototubes provide $25 \%$ photocathode coverage. A schematic of the LSND detector is shown in Fig. 1. The tank is filled with 167 metric tons of liquid scintillator consisting of mineral oil and $0.031 \mathrm{~g} / \mathrm{l}$ of $\mathrm{b}$-PBD. The low scintillator concentration allows the detection of both Cerenkov light and scintillation light and yields a relatively long attenuation length of more
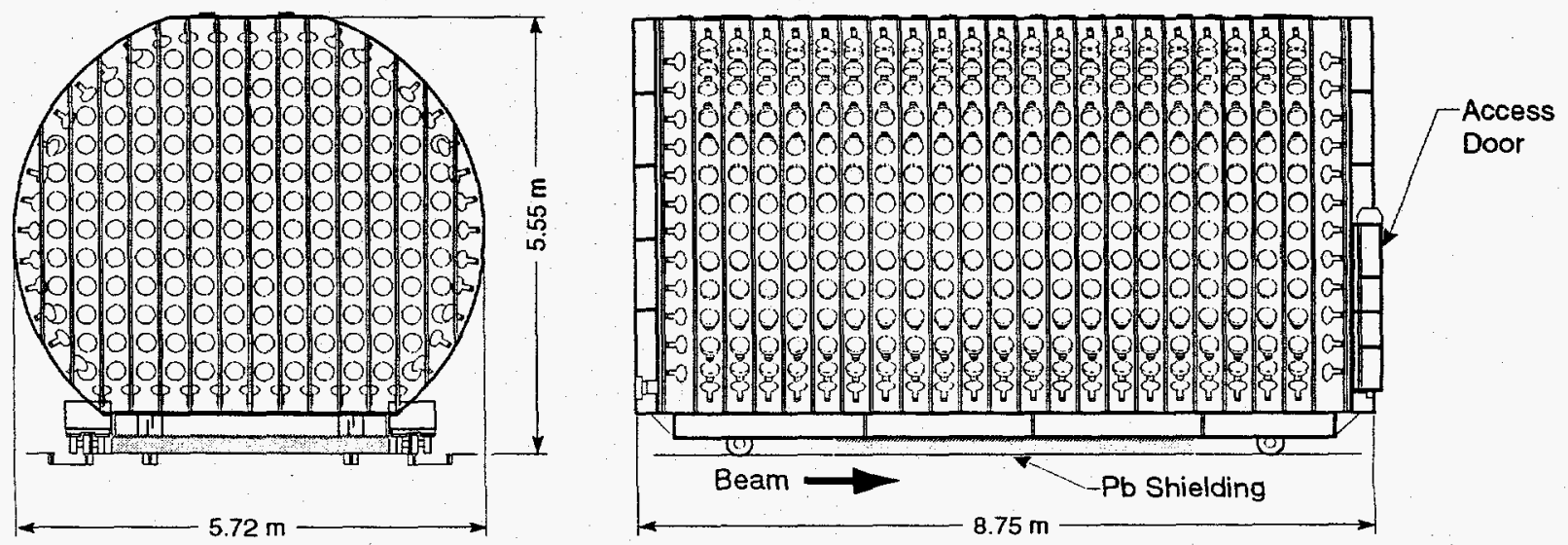

Fig. 1. A schematic of the LSND detector. 
than $20 \mathrm{~m}$ for wavelengths greater than $400 \mathrm{~nm}$. 6) A typical $45-\mathrm{MeV}$ electron created in the detector produces a total of $\sim 1500$ photoelectrons, of which $\sim 280$ photoelectrons are in the Cerenkov cone. The phototube time and pulse height signals are used to reconstruct the track with an average r.m.s. position resolution of $\sim 30 \mathrm{~cm}$, an angular resolution of $\sim 12$ degrees, and an energy resolution of $\sim 7 \%$. The Cerenkov cone for relativistic particles and the time distribution of the light, which is broader for nonrelativistic particles, give excellent particle identification. Surrounding the detector is a veto shield, ${ }^{7)}$ which tags cosmic ray muons going through the detector.

\section{DATA}

The signature for a $\bar{v}_{e}$ interaction in the detector is the reaction $\bar{v}_{e} p \rightarrow e^{+} n$ followed by $n p \rightarrow d \gamma(2.2 \mathrm{MeV})$. A likelihood ratio, $R$, is employed to determine whether a $\gamma$ is a $2.2-\mathrm{MeV}$ photon correlated with a positron or is from an accidental coincidence. $R$ is the likelihood that the $\gamma$ is correlated, divided by the likelihood that it is accidental. $R$ depends on the number of hit phototubes for the $\gamma$, the reconstructed distance between the positron and the $\gamma$, and the relative time between the $\gamma$ and positron. Figure 2 shows the $R$ distribution, beam-on minus beam-off, for events with positrons in the $36<E<60 \mathrm{MeV}$ energy range. The dashed histogram is the result of the $R$ fit for events without a recoil neutron, and the solid histogram is the total fit, including events with a neutron. After subtracting the neutrino background with a recoil neutron there is a total excess of $51.8_{-16.9}^{+18.7} \pm 8.0$ events, which if due to neutrino oscillations corresponds to an oscillation probability of $(0.31 \pm 0.12 \pm 0.05) \%$.

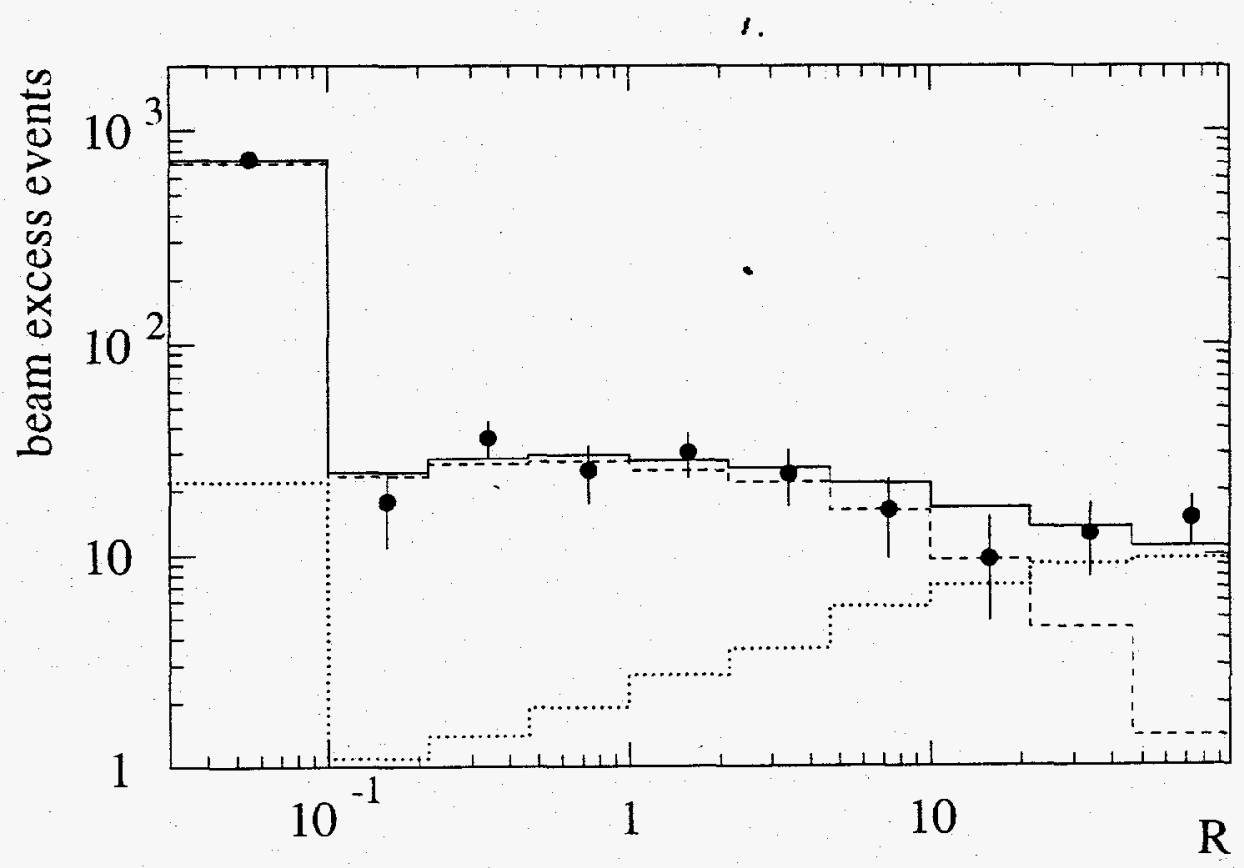

Fig. 2. The $R$ distribution, beam-on minus beam-off excess, for events that have energies in the range $20<E_{e}<60 \mathrm{MeV}$. The solid curve is the best fit to the data, while the dashed curve is the component of the fit with an uncorrelated $\gamma$. 

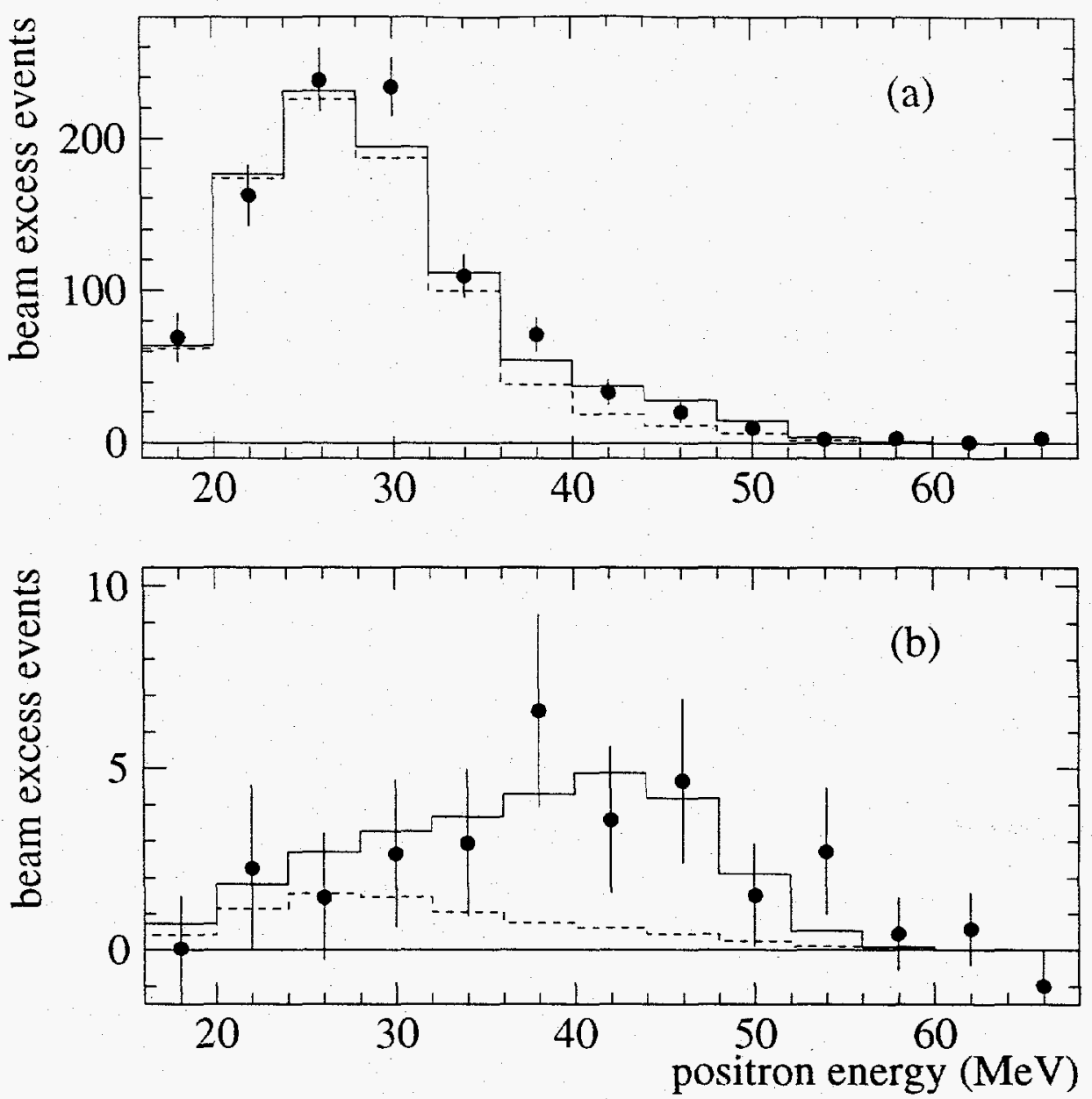

Fig. 3. The energy distribution for events with (a) $R \geq 0$ and (b) $R>30$. Shown in the figure are the beam-excess data, estimated neutrino background (dashed), and expected distribution for neutrino oscillations at large $\Delta m_{l}^{2}$. plus estimated neutrino background (solid).

Figure 3 shows the electron energy distribution, beam-on minus beam-off excess, for events (a) without a $\gamma$ requirement and (b) events with an associated $\gamma$ with $R>30$. For this latter requirement, the total $2.2-\mathrm{MeV} \gamma$ detection efficiency is $23 \%$ and the probability that an event has an accidental $\gamma$ in coincidence is $0.6 \%$. The dashed histogram shows the background from expected neutrino interactions. There are 22 events beam-on in the $36<E<60 \mathrm{MeV}$ energy range and a total estimated background (beam-off plus neutrino-induced background) of $4.6 \pm 0.6$ events. The probability that this excess is a statistical fluctuation is $<10^{-7}$. Table 1 gives the background estimate for events in the $36<E_{e}<60 \mathrm{MeV}$ energy range with $R \geq 0$ and $R>$ 30. Figure 4 shows the spatial distributions for the beam on-off excess events with $R \geq$ 0 and $R>30$, while Fig. 5 shows the $\cos \theta_{b}$, distribution for the $R>30$ sample, where $\theta_{b}$ is the angle between the neutrino direction and the reconstructed positron direction. The observed average value of $\cos \theta_{b}$ is $0.20 \pm 0.13$, in agreement with the expected value of 0.16 for $\bar{v}_{c} p$ interactions. Also, Fig. 6 shows the associated $\gamma$ time, 
Table 1. A list of all backgrounds with the expected number of background events in the $36<E_{e}<60 \mathrm{MeV}$ energy range for $R \geq 0$ and $R>30$. The neutrinos are from either $\pi$ and $\mu$ decay at rest (DAR) or decay in flight (DIF). Also shown are the number of events expected for $100 \% \bar{v}_{\mu} \rightarrow \bar{v}_{e}$ transmutation.

\begin{tabular}{|c|c|c|c|}
\hline Background & Neutrino Source & $\begin{array}{c}\text { Events with } \\
R \geq 0\end{array}$ & $\begin{array}{c}\text { Events with } \\
\qquad R>30\end{array}$ \\
\hline Beam Off & & $146.5 \pm 3.2$ & $2.52 \pm 0.42$ \\
\hline Beam-Related Neutrons & & $<0.7$ & $<0.1$ \\
\hline $\bar{v}_{e} p \rightarrow e^{+} n$ & $\mu^{-} \rightarrow e^{--} v_{\mu} \bar{v}_{e} \mathrm{DAR}$ & $4.8 \pm 1.0$ & $1.10 \pm 0.22$ \\
\hline $\bar{v}_{\mu} p \rightarrow \mu^{+} n$ & $\pi^{-} \rightarrow \mu^{-} \bar{v}_{\mu}$ DIF & $2.7 \pm 1.3$ & $0.62 \pm 0.31$ \\
\hline $\bar{v}_{e} p \rightarrow e^{+} n$ & $\pi \rightarrow e v$ and $\mu \rightarrow e v \bar{v}$ DIF & $0.1 \pm 0.1$ & 0 \\
\hline Total with Neutrons & & $7.6 \pm 1.6$ & $1.72 \pm 0.38$ \\
\hline$v_{\mu} \mathrm{C} \rightarrow \mu^{-} X$ & $\pi^{+} \rightarrow \mu^{+} v_{\mu}$ DIF & $8.1 \pm 4.0$ & $0.05 \pm 0.02$ \\
\hline$v_{e}{ }^{12} \mathrm{C} \rightarrow e^{-12} \mathrm{~N}$ & $\mu^{+} \rightarrow e^{+} \bar{v}_{\mu} v_{e}$ DAR & $20.1 \pm 4.0$ & $0.12 \pm 0.02$ \\
\hline$v_{e}{ }^{13} \mathrm{C} \rightarrow e^{-13} \mathrm{~N}$ & $\mu^{+} \rightarrow e^{+} \bar{v}_{\mu} v_{\mathcal{e}}$ DAR & $22.5 \pm 4.5$ & $0.14 \pm 0.03$ \\
\hline$v e \rightarrow v e$ & $\mu^{+} \rightarrow e^{+} \bar{v}_{\mu} v_{e}$ DAR & $12.0 \pm 1.2$ & $0.07 \pm 0.01$ \\
\hline$v e \rightarrow v e$ & $\pi \rightarrow \mu v_{\mu}$ DIF & $1.5 \pm 0.3$ & $0.01 \pm 0.01$ \\
\hline$v_{e} \mathrm{C} \rightarrow e^{-} X$ & $\pi \rightarrow e v_{e}$ DAR & $3.6 \pm 0.7$ & $0.02 \pm 0.01$ \\
\hline$v_{\mu} \mathrm{C} \rightarrow \pi \mathrm{X}$ & $\pi \rightarrow \mu v_{\mu}$ DIF & $0.2 \pm 0.1$ & 0 \\
\hline$v_{e} \mathrm{C} \rightarrow e^{-} \mathrm{X}$ & $\pi \rightarrow e v$ and $\mu \rightarrow e v \bar{v}$ DIF & $0.6 \pm 0.1$ & 0 \\
\hline Total without Neutrons & & $68.6 \pm 7.4$ & $0.41 \pm 0.04$ \\
\hline Grand Total & & $222.7 \pm 8.2$ & $4.65 \pm 0.57$ \\
\hline $100 \%$ Transmutation & $\mu^{+} \rightarrow e^{+} \bar{v}_{\mu} v_{e}$ DAR & $12500 \pm 1250$ & $2875 \pm 345$ \\
\hline
\end{tabular}

PMT hits, and distance distributions and that expected for correlated $2.2-\mathrm{MeV} \gamma \mathrm{s}$. If the observed excess is due to neutrino oscillations, Fig. 7 shows the allowed region ( $90 \%$ and $99 \%$ likelihood regions) of $\sin ^{2} 2 \theta$ vs. $\Delta m^{2}$ from a maximum likelihood fit to the $L / E$ distribution of the 22 beam-on events. Some of the allowed region is excluded by the ongoing KARMEN experiment at ISIS, ${ }^{8)}$ the E776 experiment at $B N L,{ }^{9)}$ and the Bugey reactor experiment. ${ }^{10)}$

\section{CONCLUSION}

In summary, the LSND experiment observes an excess of events with positrons in the $36<E<60 \mathrm{MeV}$ energy range that are correlated in time and space with a low energy $\gamma$. If the observed excess is interpreted as $\bar{v}_{\mu} \rightarrow \bar{v}_{\boldsymbol{e}}$ oscillations, it corresponds to an oscillation probability of $(0.31 \pm 0.12 \pm 0.05 \%)$ for the allowed regions shown in 

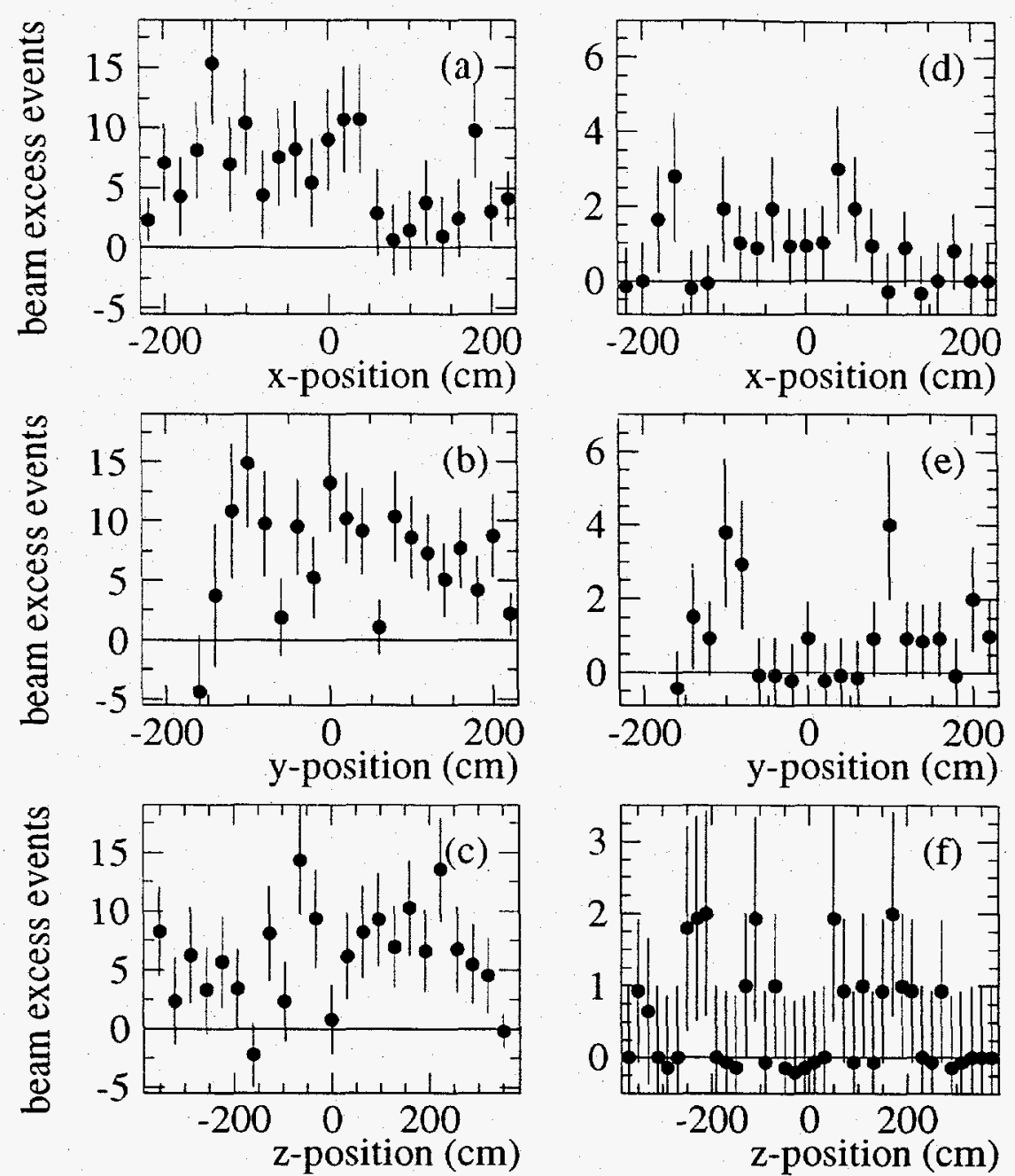

Fig. 4. The spatial distributions for beam-excess data events with $36<E_{e}<60 \mathrm{MeV}$. (a)-(c) are for $R \geq 0$ and (d)-(f) are for $R>30$.

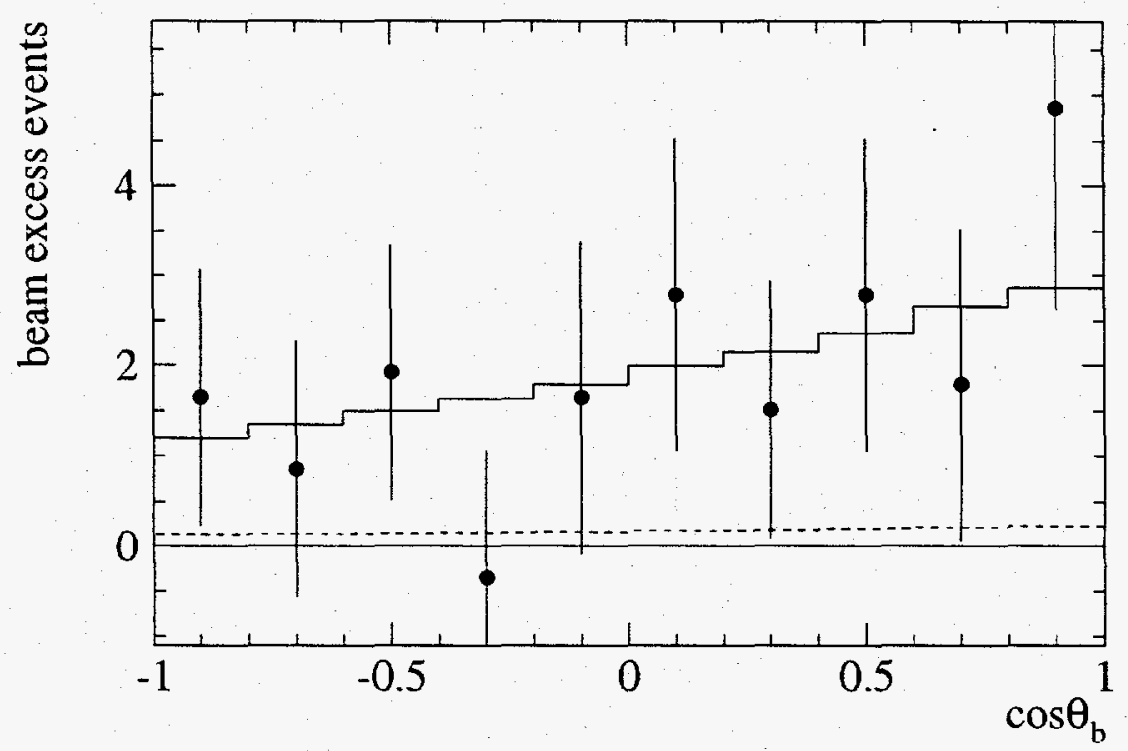

Fig. 5. The $\cos \theta_{b}$ distribution for beam-excess data events with $36<E_{e}<60 \mathrm{MeV}$ and $R>30$ and that expected for neutrino oscillations at large $\Delta m^{2}$ (solid). The dashed curve is the estimated neutrino background. $\theta_{b}$ is the $e^{+}$angle with respect to the neutrino direction. 

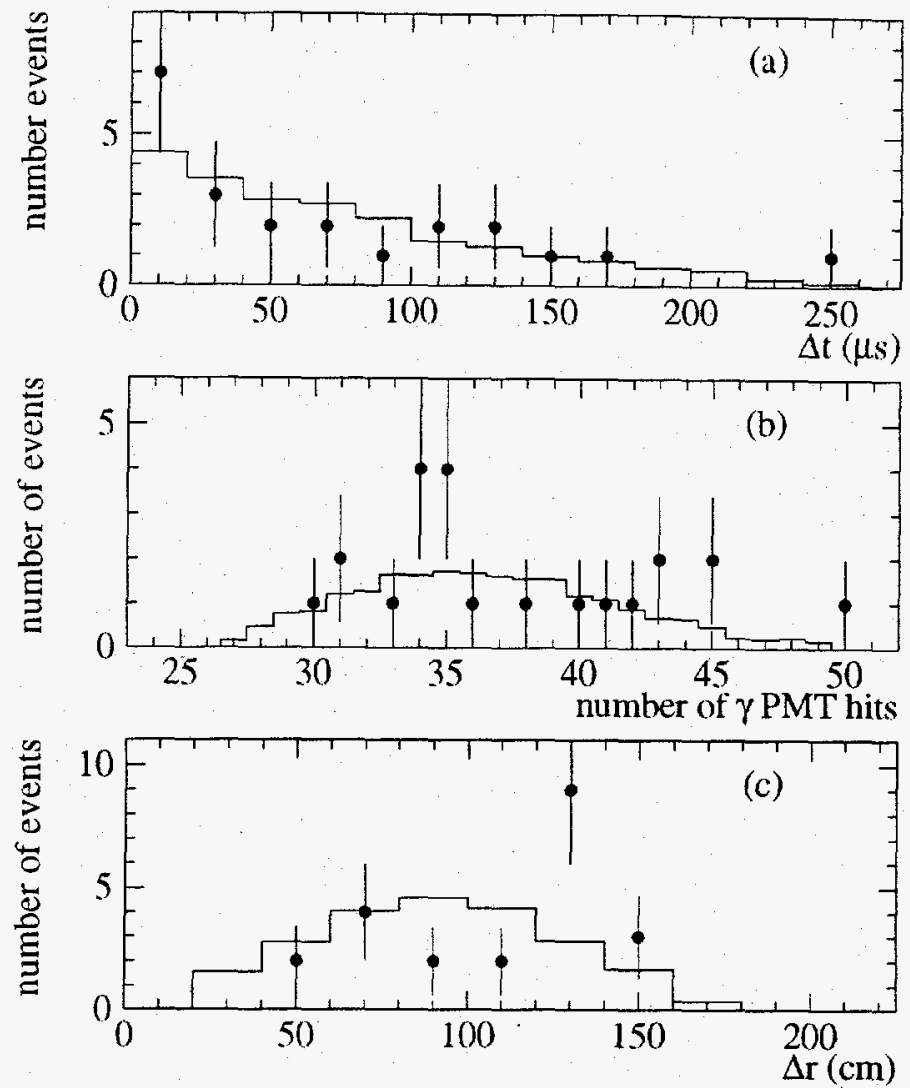

Fig. 6. The associated $\gamma$ (a) time, (b) PMT hits, and (c) distance distributions for beamon data events with $36<E_{e}<60 \mathrm{MeV}$ and $R>30$ and that expected for correlated 2.2$\mathrm{MeV} \gamma \mathrm{s}$ (solid).

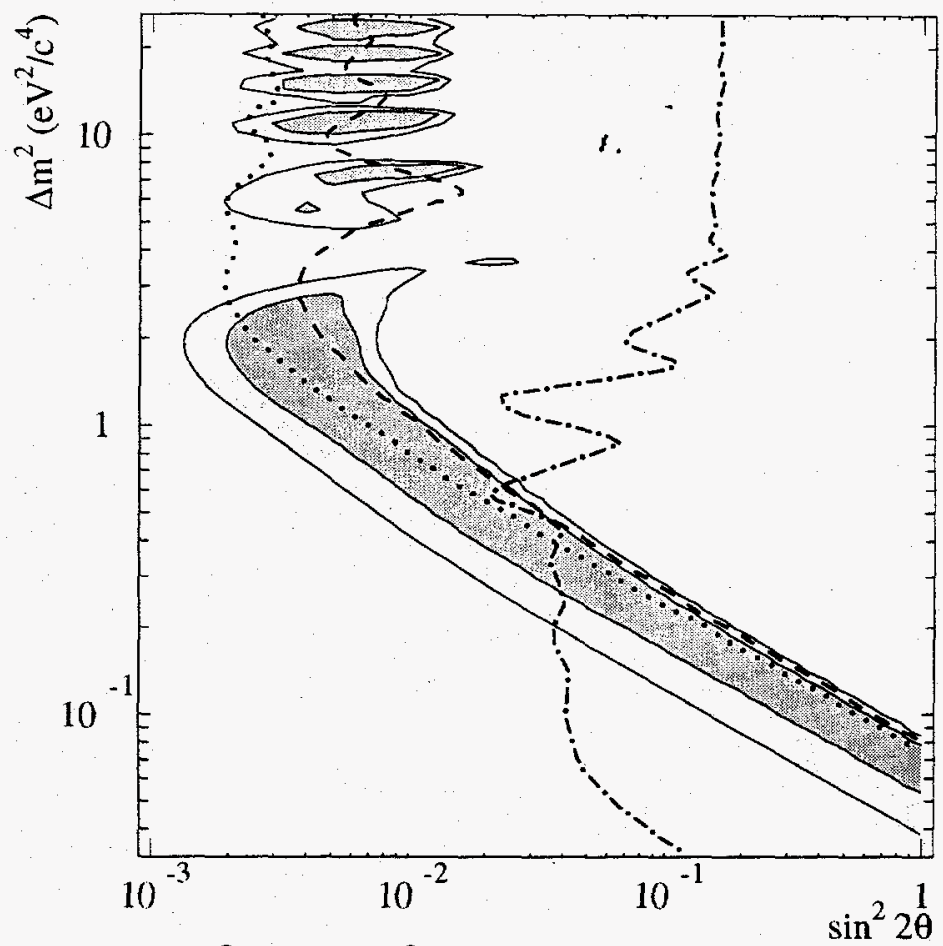

Fig. 7. Plot of the LSND $\Delta m^{2}$ vs. $\sin ^{2} 2 \theta$ favored regions. They correspond to $90 \%$ and $99 \%$ likelihood regions after the inclusion of the effects of systematic errors. Also shown are $90 \%$ C.L. limits from KARMEN at ISIS (dashed curve), E776 at BNL (dotted curve), and the Bugey reactor experiment (dot-dashed curve). 
Fig. 7. More data taking is planned for the experiment, and the performance of the detector is under continuous study. Both of these efforts are expected to improve the understanding of the phenomena described here. If neutrino oscillations have in fact been observed, then the minimal standard model would need to be modified and neutrinos would have mass sufficient to influence cosmology and the evolution of the universe.

\section{REFERENCES}

1. The LSND Collaboration consists of the following people and institutions: $K$. McIlhany, I. Stancu, W. Strossman, G. J. VanDalen (Univ. of California, Riverside); W. Vernon (Univ. of California, San Diego and IIRPA); D. O. Caldwell, M. Gray, S. Yellin (Univ. of California, Santa Barbara); D. Smith, J, Waltz (Embry-Riddle Aeronautical Univ.); A. M. Eisner, Y-X. Wang (Univ. of California IIRPA); I. Cohen (Linfield College); R. L. Burman, J. B. Donahue, F. J. Federspiel, G. T. Garvey, W. C. Louis, G. B. Mills, V. Sandberg, R. Tayloe, D. H. White (Los Alamos National Laboratory); R. M. Gunasingha, R. Imlay, H. J. Kim, W. Metcalf (Louisiana State Univ.): K. Johnston (Louisiana Tech Univ.); B. D. Dieterle, R. A. Reeder (Univ. of New Mexico); A. Fazely (Southern Univ.); C. Athanassopoulos, L. B. Auerbach, R. Majkic, J. Margulies, D. Works, Y. Xiao (Temple Univ.).

2. C. Athanassopoulos et al., Phys. Rev. Lett. 75, 2650 (1995).

3. C. Athanassopoulos et al., to appear in Phys. Rev. C (1996).

4. C. Athanassopoulos et al., submitted to Nucl. Instrum. Methods.

5. R. L. Burman, M. E. Potter, and E. S. Smith, Nucl. Instrum. Methods A291, 621 (1990); R. L. Burman, A. C. Dodd, and P. Plischke, Nucl. Instrum. Methods in Phys. Res. A368, 416 (1996).

6. R. A. Reeder et al., Nucl. Instrum. Methods A334, 353 (1993).

7. J. J. Napolitano et al., Nucl. Instrum. Methods A274, 152 (1989).

8. B. Bodmann et al., Phys. Lett. B267, 321 (1991); B. Bodmann et al., Phys. Lett. B280, 198 (1992); B. Zeitnitz et al., Prog. Part. Nucl. Phys. 32, 351 (1994).

9. L. Borodovsky et al., Phys. Rev. Lett. 68, 274 (1992).

10. B. Achkar et al., Nucl. Phys. B434, 503 (1995). 